Copyright 2021 Society of Photo-Optical Instrumentation Engineers (SPIE). One print or electronic copy may be made for personal use only. Systematic reproduction and distribution, duplication of any material in this publication for a fee or for commercial purposes, and modification of the contents of the publication are prohibited.

This is the accepted version of the paper available at https://doi.org/10.1117/12.2589126 


\title{
Interaction methods for light field displays by means of a theater model environment
}

\author{
Mary Guindy ${ }^{\mathrm{ab}}$, Attila Barsi ${ }^{\mathrm{b}}$, Peter A. Kara ${ }^{\mathrm{c}}$, Tibor Balogh ${ }^{\mathrm{a}}$, and Aniko Simon ${ }^{\mathrm{d}}$

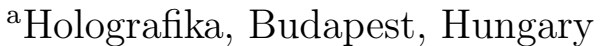 \\ ${ }^{\text {b} P a z m a n y ~ P e t e r ~ C a t h o l i c ~ U n i v e r s i t y, ~ B u d a p e s t, ~ H u n g a r y ~}$ \\ ${ }^{\mathrm{c}}$ Budapest University of Technology and Economics, Budapest, Hungary \\ dSigma Technology, Budapest, Hungary
}

\begin{abstract}
At the time of this paper, the advances in light field technology already offer 3D displays that immerse the users without the need for additional viewing devices. Despite the numerous advantages and attractive capabilities of such glasses-free 3D displays, their user interface methods are quite complicated and they are currently underwhelming when compared to conventional 2D displays, due to the fact that visual feedback can only be rendered sharply on the emission surface of light field displays. The sharp rendering of user interfaces is a necessity, as blur may hinder their fundamental functions. When it comes to 2D displays, many user interaction techniques and interfaces have been devised. Rendering a user interface on a $2 \mathrm{D}$ display could be done in various ways, such as rendering overlays on top of the rendered scene, or by using billboards. These are extensively used in modern video games. User interaction methods have proven their importance and added efficiency to virtual environments throughout the years. Due to their overall value and usefulness, interaction techniques develop immediately as new types of displays arise. With the recent advancements in visualization technologies, user interfaces have been redesigned for use in AR, VR and MR visualization. This includes on-screen augmentation, which enables interaction with visual content on the screen. Although light field displays contain immense potentials, only basic user interfaces have been devised thus far, including FOX (Focus Sliding surface), which grants users the option to scale and to rotate 3D objects. In this paper, we visualize the theater model on real light field displays and we test the different interactions by means of a monitor room. The theatre model is analogous to real-life theatres, where viewers may observe the theatrical presentation on the stage from various angles. The motivation to choose the theater model was the fact that light field visualization similarly allows multiple simultaneous viewers within its field of view, in which the content can be observed in an angledependent manner. Moreover, from the users' perspective, the theater model is thus familiar and it provides high-quality visual feedback. Furthermore, theater stages encompass a lot of interactions, including rigging and flying systems, pulleys, rotating stages, lights, curtains etc. In order to test the different interaction methods on light field displays, a theater model depicting the virtual environment was implemented. Methods for rendering the monitor room and the results of the interactions are discussed in the paper, illustrated by images of the actual visualization on light field displays. It is shown that producing plausible results with no noticeable visual artifacts is challenging, yet possible. The scientific contributions of the paper also highlight the various novel user interfaces for future light field systems and services.
\end{abstract}

Keywords: Light field, user interface, 3D rendering, light field visualization.

Further author information:

Mary Guindy: E-mail: m.guindy@holografika.com / guindy.mary.mohsen.messak@ppke.hu

Attila Barsi: E-mail: a.barsi@holografika.com

Peter A. Kara: E-mail: kara@hit.bme.hu

Tibor Balogh: E-mail: t.balogh@holografika.com

Aniko Simon: E-mail: aniko.simon@sigmatechnology.se 


\section{INTRODUCTION}

Unlike the forms of conventional 2D visualization, light field technologies represent the 3D world by multitudes of light rays. Historically, the evolution of light field visualization began in the $16^{\text {th }}$ century. ${ }^{1}$ The concept of "light field" was first defined by Leonardo Da Vinci as "radiant pyramids". ${ }^{2}$ The technical term "light field" was introduced by Gershun in 1936. ${ }^{3}$ In 1991, Adelson and Bergen described light field via the plenoptic function. ${ }^{4}$ The plenoptic function is a 7D function describing the light emitted from an object to the human eye. The main idea of the plenoptic function is to describe the intensity of light viewed from any position, for any wavelength, at any given time. The parameters of the plenoptic function are the possible eye positions $\left(V_{x}, V_{y}, V_{z}\right)$, the angle between light rays and the center of the pupil $(\theta, \phi)$, the wavelength $(\lambda)$ and the time $(t)$. Although this function provides a rather accurate description for light rays within a scene, its high dimensionality introduces complexity in calculations.

A less complex 5D representation for light fields was introduced by McMillan and Bishop. ${ }^{5}$ Furthermore, Levoy and Hanrahan reduced the dimensionality of light field representation to 4D in free spaces. ${ }^{6}$ Accordingly, rendering $4 \mathrm{D}$ light fields is an easier process and more resource-efficient.

With the evolution of modern 3D technologies, interaction techniques were developed not only for 2D, but for 3D environments as well. However, implementing such techniques on light field displays can be quite challenging. Many types of light field displays have already been developed, and all of them can be classified either as personal or collaborative devices. ${ }^{1}$ In this paper, we focus on the latter. The collaborative nature of these displays (i.e., they support a significant number of simultaneous viewers) is enabled by their wide baseline.

Our work focuses on the possible presentation models for light field displays. From these models, the theater model as well as its monitor room were implemented and tested on a wide-baseline light field display. Similarly to light field displays, theaters provide different perspectives for viewers seated in different parts of the auditorium. Additionally, theater stages offer a variety of interactions, including the rotation of stages, the usage of curtains, flying systems and many more. A monitor room depicting the system control was modelled for the light field display. Switching between both views was carried out.

The remainder of the paper is structured as follows: Section 2 reviews the 3D interaction methods. The different 3D presentation models (i.e., the specific collection of 3D interaction methods) are described in Section 3, including our own proposals. Our primary contribution, the theater model for light field visualization, is introduced in Section 4. The paper is concluded in Section 5, also pointing out potential future continuations of our work.

\section{3D INTERACTION METHODS}

Interactive 3D environments are those artificial environments or representations of real environments where users can interact with elements of the scene and navigate in real time. Interaction in such environments can be summarized in the following three tasks: ${ }^{7}$

- Navigation: changes of the view settings in the environment. In 3D applications, scenes are typically viewed via one or more view ports representing the respective (virtual) camera(s). ${ }^{7}$

- Selection and manipulation: selects a certain object in the environment and manipulates it.

- Application / System control: is not a part of the virtual environment. System control describes the usersystem communication. Moreover, it provides visual feedback where commands issued by users are used to modify the system state or the interaction method. ${ }^{8}$ This typically involves user interfaces such as overlays and menus.

The application of these 3D interaction tasks to light field displays imposes many challenges that need to be dealt with. In the remainder of this section, we discuss these limitations for horizontal-only-parallax (HOP) as well as full-parallax (FP) light field displays. 
- Navigation: Unlike conventional 3D applications, where the view settings can be easily altered based on the position of the viewer, changing the view settings for light field displays is not feasible. For HOP light field displays, it requires changing either the observer line or the set of camera positions (1D array / arc of cameras) within the scene. On the other hand, changing the view settings for FP displays requires either moving the observer rectangle or the $2 \mathrm{D}$ camera array setup. In addition to changing the observer line or the camera arrays, modifying some view settings for light field displays is not feasible either. View settings include the perspective / projection parameters, aspect ratio, resolution, near / far clipping planes and focal length. Accordingly, changing the horizontal field of view (FOV) for the horizontal parallax is not possible, whereas for FP systems, changing the FOV is not possible. Light field displays have an angularly selective nature that allows multiple viewers to view the same scene from different angles on the observer line / rectangle. Since navigation in 3D environments is mostly concerned with the view settings, the view matrix should be investigated as well. Similarly to $2 \mathrm{D}$ visualization, HOP and FP light field displays have $4 \mathrm{x} 4$ viewing matrices. Yet unlike the $2 \mathrm{D}$ scenario, where the view matrix transforms the world coordinates to camera coordinates, the view matrix of light field displays does not perform the same task. There is a 1D or 2D array of cameras in case of HOP and FP displays, respectively. Accordingly, the view matrix cannot convert world coordinates to camera coordinates due to the fact of having multiple cameras. Instead, the view matrices of light field displays convert from the world space into the region of interest (ROI) within the world space. The ROI is the area in which the objects are viewed, whereas anything outside the ROI is clipped.

- Selection and manipulation: In order to select or manipulate an object inside the environment, the object needs to be visible. For light field displays, the object under consideration must be visible from all points on the observer line or on the observer rectangle in case of HOP and FP displays, respectively. Regarding the dependability of results on the viewing angle, selection becomes unsuitable for light field displays due to their angularly selective nature. In general, a light field system is composed of multiple optical modules placed behind a semi-transparent screen. Hence, for light field displays, image space is defined for every optical module as the coordinates of its texture. ${ }^{9}$ Due to the fact that such displays have many optical modules, the selection of visualization based on image space for light field displays is impossible. Summa summarum, selection for light field displays is unattainable in image space or with dependency on the viewing angle.

- Application / System control: Light field displays act as a viewing window to the 3D world, providing 3D depth perception for the users. As a result, rendering to overlays on light field displays is not feasible, as it breaches this concept of perception. Additionally, rendering into overlays depends on the image space, and therefore, it cannot be applied to light field displays due to the aforementioned reasons. Possible alternatives to rendering into overlays include rendering to the environment or the sharp plane. The latter is preferred from the perspective of the viewers.

\section{3D PRESENTATION MODELS}

A presentation model is basically a combination of three interaction methods: (i) navigation, (ii) selection and manipulation and (iii) application / system control. Practically speaking, presentation models are used to view and arrange objects within a scene. Furthermore, they include a set of techniques for interaction and manipulation with the items present in the scene. In this section, we investigate the different 3D presentation models. This is followed by our proposal of possible presentation models for light field displays.

\subsection{Overview of 3D presentation models}

Unlike 2D interaction techniques, interaction in 3D environments is more challenging, since mapping between the $2 \mathrm{D}$ controls and the corresponding $3 \mathrm{D}$ functions is not straightforward at all. The following list contains the most relevant interaction techniques. ${ }^{7}$

- Line-up and light: All objects in the scene are lined up. A spotlight is used to focus on the main object under selection and manipulation. 
- Change focus: This technique is usually used in cinematography. It shifts the attention of spectators by changing the focus from one object / character to another. It is also known as rack focus. ${ }^{10}$

- Animation / Freezing of selected object: This technique is adopted in many video games where the selected character is being frozen / animated to indicate its selection.

- Selection halo / circle / arrow: This is one of the most common techniques in 3D video games (particularly FIFA video games ${ }^{11}$ ) where a halo / selection circle / arrow is drawn on / above the character / object under selection.

- Decals: They are used in video games where additional textures are applied over the underlying textures.

- 3D text: Self-explanatory.

- Overlays: They are used in video games to present the background graphics with rich colors. Game controllers are usually rendered into overlays in order to be visible throughout the game. ${ }^{12}$

- 3D carousels: Carousels were used extensively in the video games of the 1980s, where players were asked to enter their initials in order to record their high scores. Selecting items by means of carousels is easily understood by users, in addition to enriching to context with a sense of engagement by means of rotation. ${ }^{13}$

\subsection{Presentation models for light fields}

Unlike conventional 3D visualization, presentation models for light fields have not been investigated yet. One key point when dealing with light field presentation models is scene arrangement. Basically, arranging objects in a single row is less challenging than arranging objects along an arc or in multiple rows. Another major point in light field presentation models is the state of camera motion; whether the camera is static or dynamic. In this part of the section, we propose presentation models for light field displays.

- Navigation: Due to the various issues and challenges imposed by light field displays, it is preferable to use static cameras for scene navigation. Otherwise, objects would move back and forth between the sharp and blurry regions of visualization. In addition to static cameras, using free cameras (analogous to virtual on-the-fly cameras) is also possible.

- Selection and Manipulation: As stated in Section 2, rendering to overlays is quite difficult for light field displays. Typically, overlays are rendered on the closest plane to the observer. However, in case of light field displays, choosing the closest plane may result in blurriness due to the display optics. An alternative solution is to render on $2 \mathrm{D}$ area $(\mathrm{s})$ on the plane of the screen in order to view the overlay sharply. However, any object along the way between the $2 \mathrm{D}$ area(s) and the viewers would block the overlay. Therefore, a possible solution is to cull or to set the transparency of the objects in the occluder region in order to avoid overdraw. In addition to rendering to $2 \mathrm{D}$ area(s), rendering to $3 \mathrm{D}$ regions can actually be effective. It can be performed by using the following proposed techniques:

- Bounding box outlines: Using the axis-aligned bounding boxes (AABBs) of the objects to do the selection as drawing 2D shapes around the selected object would not work in 3D.

- Color Change: Changing the color / material (e.g., emission or light) of the selected object.

- Decals: Changing the texture of the selected object.

- Selection tube / halo / circle / arrow: see previous subsection.

- Animations: For objects being manipulated or selected by means of animation, spatial bounds should be considered.

- Hiding / Revealing: Objects in the scene are aligned in one row in the sharp region of the screen of the light field display. An extra object is used to hide all objects in the scene except for the object under selection / manipulation. Figure 1a illustrates this technique. 
- Change of object arrangement / spatial position: An example for this technique is using the line-up method, where all items of the scene are placed in one row in the blurry region of the screen. Whenever an item is selected, it moves forward / backward into the sharp region, whereas the remaining items retain their blurry states. Hence, the selected item is sharper in comparison and shall attract the attention of the viewer(s). Movement can be performed in a straight line or by means of 3D carousels. This could be applied by placing half of the carousels in the sharp region while placing the other half in the blurry region. In this case, all objects are placed in the blurry part of the carousel and rotation is applied only to the carousel holding the object under selection in order to position it in the sharp region. Figures $1 \mathrm{c}$ and $1 \mathrm{~b}$ illustrate two ways for using $3 \mathrm{D}$ carousels on light field displays. The first figure depicts the placement of all items on a single elliptical carousel where the items under selection are placed on the front part of the carousel (i.e., in the sharp region). The second figure places each item on an individual carousel. Items are positioned on the carousel in a way that they inhabit the blurry region, whereas the carousel holding the item under selection is rotated in order to place the item in the sharp region of the screen.

- Application / System control: For light field displays, system control can be achieved by rendering the user interface into $2 \mathrm{D}$ area $(\mathrm{s})$, in a way similar to that stated earlier regarding selection / manipulation. As an alternative, the separation of the main scene and the 3D controls could be performed spatially while providing feedback of the main scene on the 3D control geometry. In all the techniques used for application / system control, widget design needs to take into account visibility along the observer line / rectangle.

Table 1 introduces the possible 3D presentation models for light field displays by combining some of the aforementioned techniques to constitute plausible yet effective presentation models.

\section{THEATER MODEL FOR LIGHT FIELD VISUALIZATION}

So far, we have proposed and investigated different presentation models that could be used for light field visualization. Among these suggested models, the theater model is potentially the most efficient, and thus may provide the best visual experience. Similarly to theaters, multi-user light field displays have the same viewing experience, as they allow numerous simultaneous viewers within their FOV, in which the content can be observed in an angularly-dependent manner. In addition to allowing the effective presence of simultaneous observers, high-quality visual feedback is provided by means of a monitor control room. Furthermore, theaters encompass lots of interactions and animations for their presentation elements.

\subsection{Technical considerations}

In order to test the different interaction methods for light field displays, a proscenium theater model and a monitor room were modelled using MAYA, ${ }^{14}$ and they were visualized on the HoloVizio C80 light field display. ${ }^{15}$ The C80 has an aspect ratio of 16:9 and a 40 deg-horizontal viewing angle. Although animations and interaction methods are easily implemented for and viewed on conventional 2D displays, 3D light field displays impose some challenges and limitations. ${ }^{16}$ One of these challenges is the fact that only a certain portion of the visualization area supports sharp rendering, and thus the focus of the content is limited to that specific area.

Figure 3 shows the top view for a typical setup of light field displays. The black line depicts the screen, whereas the blue lines show the viewing angles. The dotted lines encompass the blurry regions. The area surrounding the screen is the one where objects are rendered sharply. Hence, if an object is animated on a line that is perpendicular to the observer line / rectangle, the object moves into and out of focus as it crosses the blurry and sharp areas. Accordingly, it is better to consider animations along any plane perpendicular to the screen (i.e., animations that include right / left or top / down motions). However, if animations along the lines perpendicular to the observer line / rectangle are to be considered, then they should be done within a small range in order to avoid the potential crossing. Therefore, the theater model fulfils these requirements by the animations of rigging / flying system and curtains. 


\begin{tabular}{|c|c|c|c|}
\hline Presentation model & Navigation & Selection and manipulation & $\begin{array}{l}\text { Application / System } \\
\text { control }\end{array}$ \\
\hline Line-up & Static Camera & $\begin{array}{l}\text { Bounding box outlines } \\
\text { Color change } \\
\text { Decals } \\
\text { Selection tube / halo / circle / } \\
\text { arrow } \\
\text { Animation } \\
\text { Change of object arrangement / } \\
\text { spatial position }\end{array}$ & Switch 2 scenes \\
\hline Carousel & Static camera & $\begin{array}{l}\text { Change of object arrangement / } \\
\text { spatial position }\end{array}$ & Switch 2 scenes \\
\hline 3D sphere & Static camera & $\begin{array}{l}\text { Change of object arrangement / } \\
\text { spatial position }\end{array}$ & Switch 2 scenes \\
\hline CAD / CAM & Free camera & $\mathrm{AABB}$ & $\begin{array}{l}\text { 2D areas on screen }+ \\
\text { spatial separation for } \\
\text { navigation feedback }\end{array}$ \\
\hline Medical & Orbiter camera & Select on $2 \mathrm{D}$ area $(\mathrm{s})$ & $\begin{array}{l}\text { 2D areas on screen }+ \\
\text { spatial separation for } \\
\text { navigation feedback }\end{array}$ \\
\hline Theater & Static camera & $\begin{array}{l}\text { Change colors } \\
\text { Change of object arrangement / } \\
\text { spatial position } \\
\text { Hiding / Revealing }\end{array}$ & Switch 2 scenes \\
\hline
\end{tabular}

Table 1: Presentation models for light field displays.

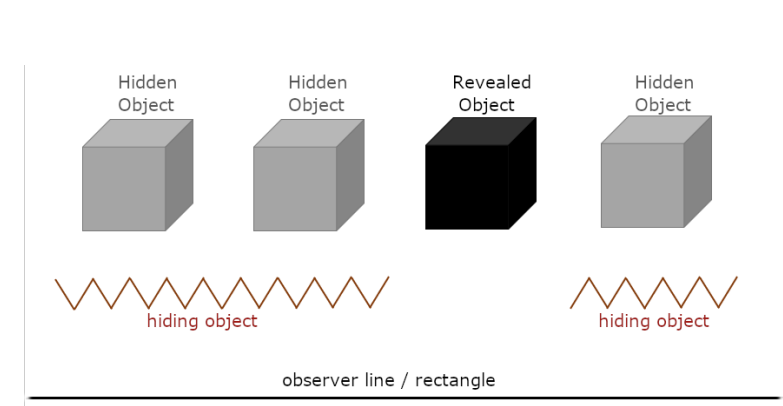

(a) Hiding / Revealing

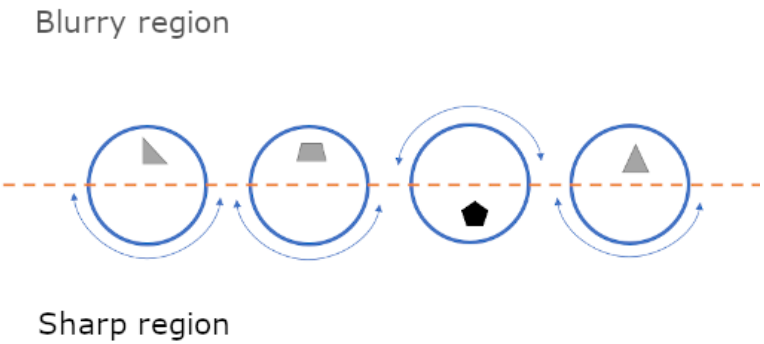

(b) 3D carousels

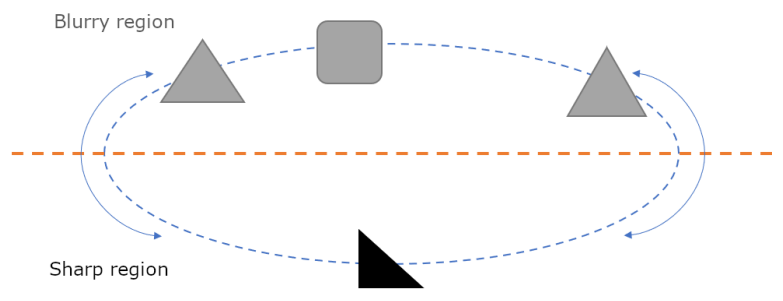

(c) Single carousel with elliptical path

Figure 1: Selection and manipulation for light field displays. 

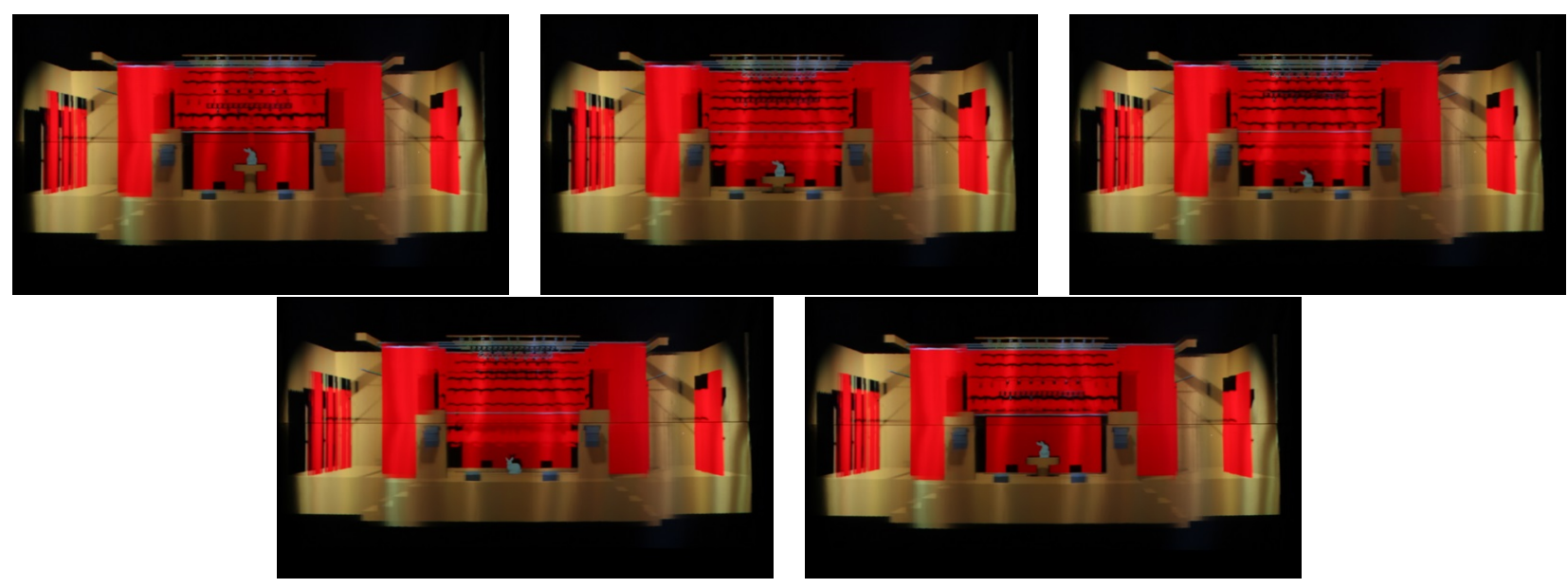

(a) Rotating stage while moving up / down
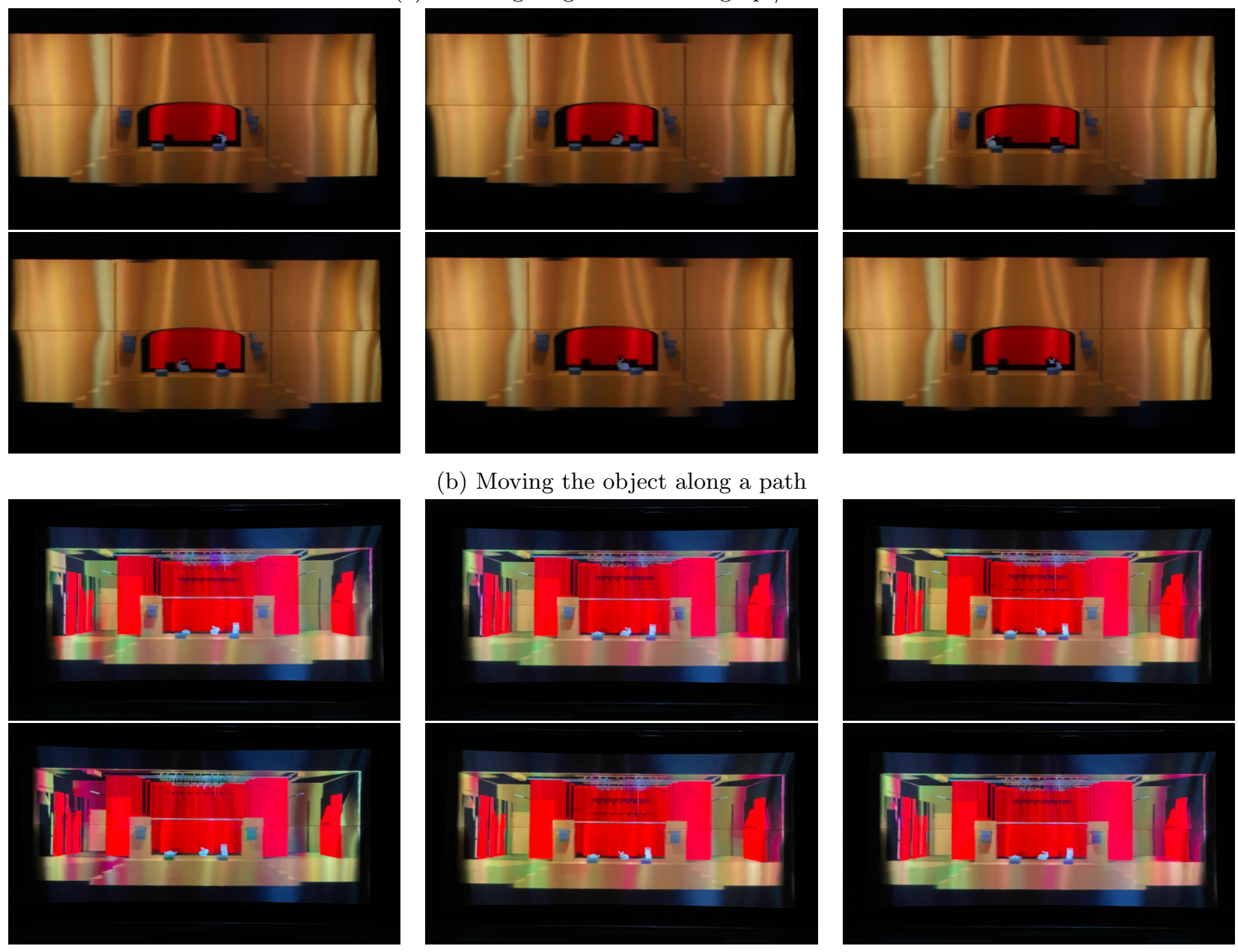

(c) Usage of 3D carousels

Figure 2: Theater model simulation on light field display. 


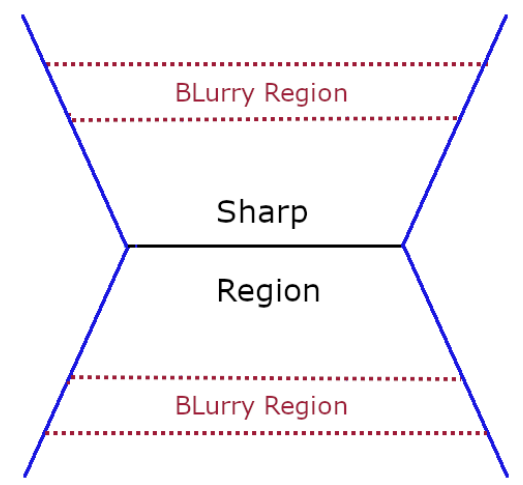

Figure 3: Top view of light field display setup.

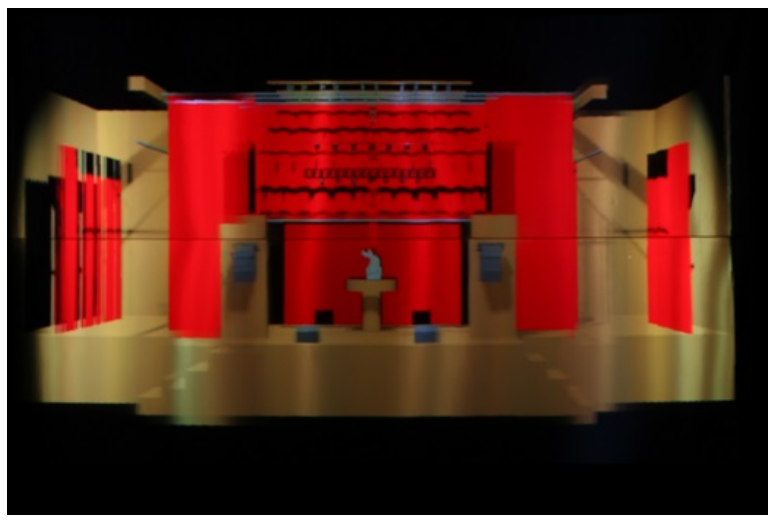

(a) Theater model

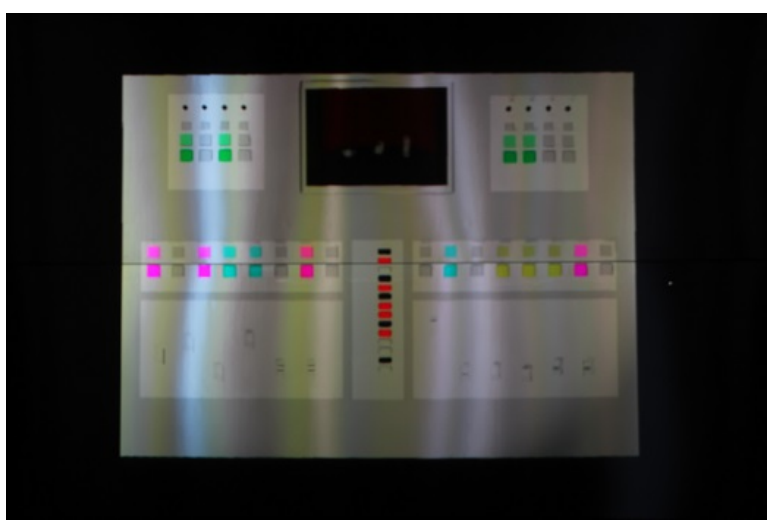

(b) Monitor control model

Figure 4: Theater and monitor room models.

\subsection{Utilization of the theater model}

As stated earlier, both the theater model and the monitor room were modelled in MAYA. The models of bunny, buddha and teapot were imported from the Computer Graphics Archive. ${ }^{17}$ Figure 4 shows the theater model and the monitor room. The monitor (control) room depicts the application / system control in the presentation model of the theater. Switching back and forth between these views is achieved by pressing buttons. The corresponding animation / lighting is activated within the theater model and the monitor room is viewed at that time with a display screen showing the current theater view. Once the corresponding animation is activated, the view switches back to the theater model. Navigation within the theater model is performed via a static camera.

\subsection{Evaluation and Results}

Figure 2 shows different images from the theater model. In our work, we tested different ideas for the selection / manipulation of objects:

- A theater model with a rotating stage, where the rotating stage is placed in the sharp region of the light field display. Hence, the movement of the theater stage in the up / down direction and rotation do not cause any blurring effects.

- A theater model with an object animated along a path to change its position.

- Using curtains to hide some elements while displaying others under selection to apply the hiding / revealing technique.

- Spatial positioning of presentation elements is done within the sharp region in a plane parallel to the screen, thus avoiding the problem of moving in and out of the sharp region (e.g., animation of curtains and rigging 
/ flying system). Animation of curtains and flying systems is done within their plane (right / left and up / down motion), hence avoiding the problem of moving in and out of the blurry region of the light field display.

- Usage of rotating stages where half of the stage is placed in the blurry region and the other half in the sharp region. Spatial positioning of objects that are selected is done within the sharp region.

- Animating the spotlights and spotlight reflectors by rotating them within a very small range and thus they do not cause an issue on the light field display.

\section{CONCLUSION}

Interaction techniques for wide-baseline light field displays is a new, yet promising research topic. In this paper, we investigated the possible presentation models for light field displays and used the theater model for illustration and testing.

As future continuation of this work, large-scale subjective tests could be carried out regarding the proposed metrics in order to get a deeper understanding of the interaction techniques that are the most suitable for light field displays.

\section{Acknowledgments}

This project has received funding from the European Union's Horizon 2020 research and innovation programme under the Marie Skłodowska-Curie grant agreement No 813170. Also received funding by 2018-2.1.3-EUREKA2018-00007 and KFI 16-1-2017-0015, NRDI Fund, Hungary.

\section{REFERENCES}

[1] Balram, N. and Tošić, I., "Light-field imaging and display systems," Information Display 32, 6-13 (2016).

[2] Richter, J. P. et al., [The notebooks of Leonardo da Vinci], vol. 2, Courier Corporation (1970).

[3] Gershun, A., "The light field," Journal of Mathematics and Physics 18(1-4), 51-151 (1939).

[4] Adelson, E. H. and Bergen, J. R., [The plenoptic function and the elements of early vision], vol. 2, Vision and Modeling Group, Media Laboratory, Massachusetts Institute of Technology (1991).

[5] McMillan, L. and Bishop, G., "Plenoptic modeling: An image-based rendering system," in [Proceedings of the 22nd Annual Conference on Computer Graphics and Interactive Techniques], SIGGRAPH '95, 39-46, Association for Computing Machinery, New York, NY, USA (1995).

[6] Levoy, M. and Hanrahan, P., "Light field rendering," in [Proceedings of the 23rd Annual Conference on Computer Graphics and Interactive Techniques], SIGGRAPH '96, 31-42, Association for Computing Machinery, New York, NY, USA (1996).

[7] Jankowski, J. and Hachet, M., "A survey of interaction techniques for interactive 3D environments," in [Eurographics 2013-STAR], (2013).

[8] Bowman, D. A., Kruijff, E., LaViola, J. J., and Poupyrev, I., "An introduction to 3-D user interface design," Presence: Teleoper. Virtual Environ. 10(1), 96-108 (2001).

[9] Doronin, O., Barsi, A., Kara, P. A., and Martini, M. G., "Ray tracing for holovizio light field displays," in [2017 International Conference on 3D Immersion (IC3D)], 1-8 (2017).

[10] Brown, B., [Cinematography: theory and practice: image making for cinematographers and directors], Taylor \& Francis (2016).

[11] Electronic Arts, "FIFA Video Games."

https://www.ea.com/en-gb/games/fifa.

[12] Guttenbrunner, M., Becker, C., and Rauber, A., "Keeping the game alive: Evaluating strategies for the preservation of console video games," (2010).

[13] Björkskog, C., Jacucci, G., Lorentin, B., and Gamberini, L., "Mobile implementation of a web 3D carousel with touch input," in [Proceedings of the 11th International Conference on Human-Computer Interaction with Mobile Devices and Services], MobileHCI '09, Association for Computing Machinery, New York, NY, USA (2009). 
[14] Autodesk, INC., "MAYA."

https : /autodesk . com/maya.

[15] Holografika, "HoloVizio C80 glasses-free 3D cinema system." https://holografika.com/c80-glasses-free-3d-cinema/.

[16] Cserkaszky, A., Kara, P. A., Tamboli, R. R., Barsi, A., Martini, M. G., and Balogh, T., "Light-field capture and display systems: limitations, challenges, and potentials," in [Novel Optical Systems Design and Optimization XXI], International Society for Optics and Photonics (2018).

[17] McGuire, M., "Computer Graphics Archive," (2017).

https://casual-effects.com/data. 